\title{
Acute Diarrheal Illness - The Most Common Medical Problem Worldwide
}

\author{
Angela Revelas \\ St.Nicolaos General Hospital ,Crete, Greece
}

Corresponding Author: Angela Revelas E mail:

donnoiko@gmail.com Received :20/7/2012 Accepted after revision: $20 / 8 / 2012$ Key words :diarrhea; Viral;bacterial; protozoal

Diarrhea can result in significant morbidity and mortality, depending on the degree of severity. The history and clinical presentation should dictate initial treatment and management.

\section{INTRODUCTION}

Acute diarrheal illness is one of the most common medical problem worldwide. It can be caused by bacteria, protozoa, or viruses and can demonstrate a wide spectrum of clinical severity. The medical history and physical examination are the keys to an effective diagnosis. Laboratory investigations and antibiotic therapies are useful in select situations, but they often do not augment effective management. Acute diarrhea is defined as the abrupt onset of abnormally high fluid content in the stool, more than the normal value of approximately $10 \mathrm{~mL} / \mathrm{Kg} / \mathrm{d}$ in the infant and young child, and more than $200 \mathrm{~g} / \mathrm{d}$ in the teenager and adult. This situation typically implies an increased frequency of bowel movements, which can range from 4-5 to more than 20 times per day. Diarrheal episodes are classically distinguished into acute and chronic based on their duration. Acute diarrhea is thus defined as an episode that has an acute onset and lasts no longer than 14 days; chronic diarrhea is defined as an episode that lasts longer than 14 days.

\section{EPIDEMIOLOGY}

Children living in developing countries have more frequent episodes of diarrhea than other children and develop intestinal immunity to the majority of enteric pathogens[1]. As a result of improved sanitation and food processing, travelers from the Western World lack this immunity and often develop diarrhea when abroad. Travelers diarrhea is usually caused by the organisms responsible for pediatric diarrhea in those areas.

The most common cause of infectious diarrhea worldwide is rotavirus[2]. Mortality from acute diarrhea is overall declining but remains high. Most estimates have diarrhea as the second cause of childhood mortality, with $18 \%$ of the 10,6 million yearly deaths in children younger than age 5 years. In countries where the toll of diarrhea is highest, poverty also adds an enormous additional burden, and long-term consequences of the vicious cycle of enteric infections, diarrhea, and malnutrition are devastating. Most cases of infectious diarrhea are not sex specific. Females have a higher incidence of campylobacter species infections and hemolytic uremic syndrome. Viral diarrhea is most common in young children. Rotavirus and Adenovirus are particularly prevalent in children younger than 2 years[3].Astrovirus and Norovirus usually infect children younger than 5 years. Yersinia enterocolitis typically infects children 
younger than 1 year, and the Aeromonas organism is a significant cause of diarrhea in young children. Very young children are particularly susceptible to secondary dehydration [4] and secondary nutrient malabsorption. Age and nutritional status appear to be the most important host factors in determining the severity and the duration of diarrhea.The younger the child, the higher is the risk for severe, lifethreatening dehydration as a result of the high body- water turnover and limited renal compensatory capacity of very young children.

\section{PATHOLOGY}

The pathogens or toxins that produce acute infectious diarrhea are usually ingested unknowingly. Inadequate sanitation and poor hygiene figure prominently in many patients with diarrhea. Defence mechanisms, such as the acidic $\mathrm{pH}$ of the stomach, rapid transit time of the small bowel, and antibodies produced in the in the jejunum and proximal ileum reduce number of microorganisms. The ileocecal valve normally prevents the movement of colonic bacteria into the small bowel; individuals with a deficiency in any of these processes are at increased risk of developing diarrhea. Pathogenic organisms can pass through the acidic milieu of the stomach if they are acid-resistant, such as Shigella. Massive ingestion of contaminated food or water can also yield an adequate number of viable organisms. Food can neutralize acid locally and provide a haven for microorganisms. The organisms that survive and reach the small bowel, such as E.coli or Vibrio cholerae, can colonize the area and cause diarrhea. Mucosal invasion is another pathogenic mechanism and occurs with Salmonella sp, Shigella, and Campylobacter jejuni. Passage into the colon allows organisms, such as Salmonellae, to invade the local mucosa. Enteric pathogens or their toxins can cause diarrhea either by increasing small intestinal secretions beyond the colon's absorptive capacity or by impairing the colon's absorptive capacity directly.

\section{DIAGNOSIS}

Diarrhea is most commonly due to viral gastroenteritis with Rotavirus, which accounts for $40 \%$ of cases in children under five [5]. In travelers however bacterial infectious predominate [6]. Various toxins such as mushroom poisoning and drugs can also cause acute diarrhea. Chronic diarrhea can be the part of the presentations of a number of chronic medical conditions affecting the intestine.Common causes include ulcerative colitis, Crohn's disease, microscopic colitis, celiac disease, irritable bowel syndrome, and bile acid malabsorption.Norovirus is the most common cause of viral diarrhea in adults [7] but Rotavirus is the most common cause in children under five years old [8]. Adenovirus types 40 and 41[9] and Astroviruses cause a significant number of infections [10]. The bacterium Campylobacter is a common cause of bacterial diarrhea, but infections by Salmonellae, Shigellae and some strains of Esherichia coli are frequent [11]. In the elderly, particularly those who have been treated with antibiotics for unrelated infections, a toxin produced by Clostridium difficile, often causes severe diarrhea [12]. Parasites do not often cause diarrhea except for the protozoan Giardia, which can cause chronic infections if these are not diagnosed and treated with drugs such as metronidazole [13] and Entamoeba histolytica [14,15]. Other infectious agents such as parasites and bacterial toxins also occur [6]. In sanitary living conditions where there is ample food and a supply of clean water, an otherwise healthy person usually recovers from viral infections in a few days. However, for ill or malnourished individuals, diarrhea can lead to severe dehydration, and can become life-threatening [16].

\section{LABORATORY STUDIES}

The following may be noted in patients with diarrhea.

In patients with diarrhea, a stool $\mathrm{pH}$ level of 5.5 or less or presence of reducing substances indicates carbohydrate intolerance, which is usually secondary to viral illness and transient in nature.

Enteroinvasive infections of the large bowel produce leukocytes, predominantly neutrophils, to be shed into stool. Absence of fecal leukocytes does not eliminate the possibility of enteroinvasive organisms. However, presence of fecal leukocytes eliminates consideration of enterotoxigenic E.coli, Vibrio species, and viruses.

Examine any exudates found in stool for leukocytes. Such exudates highly suggest colitis. $80 \%$ positive predictive value colitis can be infectious, allergic, or part of inflammatory 
bowel disease (Crohn's disease, ulcerative colitis).

Many different culture mediums are used to isolate bacteria.

With stool not cultured within 2 hours of collection, refrigerate at $40 \mathrm{C}$ or place in a transport medium. Although stool cultures are useful when positive, yield is low.

Always culture stool for Salmonella, Shigella, and Campylobacter organisms and Yesterocolitica in the presence of clinical signs of colitis or if fecal leucocytes are found.

Look for C.difficile in persons with episodes of diarrhea characterized by colitis and or blood in the stools. Remember that acute-onset diarrheal episodes associated with $C$. difficile may also occur without a history of antibiotic use.

Bloody diarrhea with a history of ground beef ingestion must raise suspicion for enterhemorrhagic E.coli. If E.coli is found in the stool, determine if the type of E.coli is 0157 H7. This type of E. coli is the most common, but not only, cause of hemolytic uremic syndrome (HUS).

History of raw seafood ingestion or foreign travel should prompt additional screening for Vibrio and plesiomonas species.

\section{DIARRHEA SYNDROMES}

Gastroenteritis, as a result of food contamination is a common cause of acute infectious diarrhea. Only severe clinical presentation or the threat of botulism requires hospitalization.

Traveler's Diarrhea, The spectrum of traveler's diarrhea is primarily the result of enteric pathogens contracted orally while visiting a foreign country. This syndrome is primarily caused by enterotoxigenic E.coli. The illness is usually benign and self-limited, but it may have severe presentations with dehydration or bloody diarrhea.

Diarrhea in patients with AIDS, Although diarrhea in patients with AIDS is a broad topic, the same principles of treating acute diarrhea apply. C.difficile is commonly found in patients with AIDS because of increased hospital exposure and frequent antibiotic use .

\section{INITIAL TREATMENT OR PROGNOSIS}

In general, most causes of acute diarrhea will last only one week to ten days, especially when the patient receives specific therapy. When the diarrheal illness persists for more than two weeks, the differential diagnosis may include lactose deficiency, which can be produced by small bowel pathogens, including viral agents, enterotoxigenic $E$ coli or G.lamblia. In many cases of diarrhea, replacing lost fluid therapy, or, in severe cases, intravenously. Diet restrictions such as the BRAT diet are no longer recommended [1]. Research does not support the limiting of milk to children as doing so has no effect on duration of diarrhea.

\section{ANTIBIOTICS}

While antibiotics are beneficial in certain types of acute diarrhea, they are usually not used except in specific situations [17]. There are concerns that antibiotics may increase the risk of hemolytic uremic syndrome in people infected with Escherichia coli 0157. H7 [18]

\section{BISMUTH COMPOUNDS}

While bismuth compounds (pepto- Bismol) decreased the number of bowel movements in those with traveler's diarrhea, they do not decrease the length of illness [19]. These agents should only be used if bloody diarrhea is not present [20].

\section{BILE ACID SEQUESTRANTS}

Bile acid sequestrates such as cholestyramine, colestipol and colesevelam can be effective in chronic diarrhea due to bile acid malabsorption. Therapeutic trials of these drugs are indicated in chronic diarrhea if bile acid malabsorption cannot be diagnosed with a specific test, such as SeHCAT retention.

\section{CONCLUSION}

Acute diarrheal illness is one of the most common medical problems worldwide. The viral, bacterial, and protozoal causes of diarrhea can result in significant morbidity and mortality, depending on the degree of severity. The history and clinical presentation should dictate the initial treatment and management. The majority of patients will require only volume repletion until the diarrheal process resolves. Extensive diagnostic evaluation should be 
reserved for patients with moderate-to-severe acute or chronic illness. This approach will result in a higher diagnostic yield and more specific and cost-effective treatment.

\section{REFERENCES}

1. King CK, Glass R, Bresee JS, Duggan C. Managing acute gastroenteritis among children; oral rehydration, maintenance, and nutritional therapy. MMWR Recomm Rep 2003; 52 [1]:1-16

2. Bryce J, Boschi-Pinto C, Shibuya K, Black RE. WHO estimates of the couses of death in children. Lancet 2005; 365(9465):1147-52

3. Coffin SE, Elser J, Marchant C. Sawyer M, Pollara B, Fayorsey R, et al. Impact of acute Rotavirus gastroenteritis on pediatric outpatient practices in the United States. Pediatr Infect Dis J2006; 25(6): 489-93

4. Bellemare S, Hartling L, Wiebe N, Russell K, Craig WR, McConnell D, Klassen TP .Oral rehydration versus intravenous therapy for treating dehydration due to gastroenteritis in children:a meta-analysis of randomized controlled trials. BMC 2004;2(1):11

5. World Health Organization Global health risks: mortality and burden of disease attributable to selected major risks. WHO 2009; p 16-17.

6. Wilson ME. Diarrhea in nontravelers: risk and etiology. Clin Infect Dis 2005; 41(Suppl. 8): S541-6

7. Patel MM, Hall AJ, Vinje J, Parashar UD. Noroviruses: a comprehensive review. J Clin Virol 2009;44(1):1-8.

8. Greenberg HB, Estes MK. Rotaviruses: from pathogenesis to vaccination. Gastroenterology 2009; 136(6):1939-51.

9. Uhnoo I, Svensson L, Wadell G. Enteric Adenoviruses. Baillieres Clin Gastroenterol 1990;4:627-42

10. Mitchell DK. "Astrovirus gastroenteritis". The Pediatric Infectious Disease Journal 2000; 21 (11): $1067-9$.
11. Viswanathan UK, Hodges K, Hecht G. Enteric infection meets intestinal function: how bacterial pathogens cause diarrhoea. Nat Rev Microbiol; 2009 ; 7(2):110-9.

12. Rupnik M, Wilcox MH, Gerding DN. Clostridium difficile infection: new developments in epidemiology and pathogenesis. Nat Rev Microbiol 2009; 7(7):526-36.

13. Kiser JD, Paulson CP, Brown C. Clinical inquires :What is the most effective treatment of giardiasis. The Journal of Family practice 2008;57(4) 270-2

14. Dans L, Martínez E. "Amoebic dysentery".Clinical Evidence2006 (15): 1007-13.

15. Gonzales ML, Dans LF, Martinez EG. Antiamoebic drugs for treating amoebic colitis. Cochrane Database Syst Rev 2009;(2):CD006085.

16. Alam NH, Ashraf H. Treatment of infectious diarrhea in children. Paediatr Drugs 2003;5(3):151-65.

17. Dryden M.S., Gabb R.J., Wright S.K. Empirical treatment of severe acute community-acquired gastroenteritis with ciprofloxacin. Clin Infect Dis 1996; 22: 1019-25.

18. Wong CS, Jelacic S, Habeeb RL, Watkins SL, Tarr PI. The risk of the hemolytic-uremic syndrome after antibiotic treatment of Escherichia coli O157:H7 infections.$N$ Engl $J$ Med 2000;342(26):1930-6.

19. DuPont HL, Ericsson CD, Farthing MJ . Gorbach S, Pickering LK, Rombo L, Steffen R, Weinke T.Expert review of the evidence base for self-therapy of travelers' diarrhea. J Travel Med 2009;16(3):161-71.

20. Pawlowski SW, Warren CA, Guerrant R. Diagnosis and treatment of acute or persistent diarrhea. Gastroenterology 2009; 136(6):187486. 